\title{
Geometry and magnetic-field-induced vortices and antivortices in mesoscopic two-dimensional systems
}

\author{
A. F. Slachmuylders, ${ }^{*}$ B. Partoens ${ }^{\dagger}$ and F. M. Peeters ${ }^{\dagger}$ \\ Departement Fysica, Universiteit Antwerpen (Campus Middelheim) Groenenborgerlaan 171, B-2020 Antwerpen, Belgium
}

(Received 13 January 2005; revised manuscript received 25 February 2005; published 6 June 2005)

\begin{abstract}
A two-dimensional mesoscopic system of arbitrary shape placed in a perpendicular homogeneous magnetic field is investigated for arbitrary boundary conditions. The energy spectrum of quantum dots and the phase boundary of mesoscopic superconductors are obtained. The wave function for quantum dots and the complex order parameter for mesoscopic superconductors exhibit the simultaneous stabilization of vortices and antivortices at specific ranges of the magnetic field for noncircular symmetric samples. When the vorticity is changed, we found that antivortices can be introduced into the system in order to preserve the symmetry of the sample in the vortex pattern.
\end{abstract}

DOI: 10.1103/PhysRevB.71.245405

PACS number(s): 73.21.La, 74.78.Na

\section{INTRODUCTION}

The rapid development of nanostructuring in the last decades has made it possible to achieve important scientific and technological advances. Modern processing techniques allow the creation of mesoscopic two-dimensional (2D) systems which has led to a very rich variety of new physical phenomena. ${ }^{1}$ Here we will show that additional theoretical insight in these 2D systems can be gained by solving its associated Schrödinger equation with different boundary conditions.

On the one hand, solving the 2D Schrödinger equation with Dirichlet boundary conditions enables us to study a single electron in a quantum dot (QD), the man-made equivalent of an atom. We consider semiconductor QDs where the lateral confinement energy is much smaller than the perpendicular confinement so that we may assume that effectively the electron is moving in two dimensions. This situation corresponds to a quantum billiard model extensively studied experimentally and theoretically., ${ }^{2,3}$ Not only the possible applications of QDs (such as transistors, lasers, etc.), but also the fact that the properties of these "designer atoms" can be changed in a controlled way by electrostatic gates, changes in the dot geometry, or applied magnetic field, make them an interesting study object to understand more about the quantum world. ${ }^{4}$

On the other hand a similar differential equation describes mesoscopic superconductors. Superconductors are in the mesoscopic regime when the size of the sample becomes comparable to the penetration depth $\lambda(T)$ or the coherence length $\xi(T)$, where $\lambda(T)$ and $\xi(T)$ are typical length scales of the Ginzburg-Landau (GL) theory. ${ }^{5-7}$ Here we use the linearized GL equation to study the superconducting-normal phase boundary of the superconductors. The linearized GL equation is formally equivalent to the Schrödinger equation for a free particle, except for the boundary condition: we have to solve the Schrödinger equation but now with Von Neumann boundary conditions. The 2D Schrödinger equation with Von Neumann boundary conditions describes not only superconducting wires but also thin superconducting samples as considered in Ref. 8 for a small and thin superconducting square. We investigate the possibility of influencing the critical parameters (critical temperature and critical magnetic field) of the superconductor by manipulating the boundary conditions. These critical parameters determine whether or not the material is in the superconducting state and therefore by manipulating those boundary conditions it is possible to reach higher critical magnetic fields and higher critical temperatures.

In this paper we combine the study of both systems, put an emphasis on the differences and resemblances of both physical systems, and concentrate on the appearance and disappearance of vortices and antivortices as function of the magnetic field. Furthermore, we generalize previous works $^{8-10}$ and consider arbitrary boundary conditions and arbitrary potential heights. Of particular interest to us are the vortex patterns in mesoscopic 2D systems which are formed when a perpendicular magnetic field is present. A vortex can be defined as a flow with closed streamlines with a phase singularity in the center of it. In a quantum mechanical picture, this means that the wave function in the center of a vortex must be zero, since the wave function has to be a single-valued function. Vortices, which are a well-known phenomenon in nature, can be found on different scales, e.g., at the cosmic level (galaxies) but also on the atomic level. We will find that vortices and antivortices appear both in the QD and in superconducting systems.

The vortices in quantum mechanical systems reveal important information about the system. As the wave function is zero in the center of a vortex, the vortex pattern in QDs indicates the highest probability to find an extra electron if it would be added to the system. The pattern of vortices is therefore closely related to the correlations between electrons in a multi-electron QD. ${ }^{11,12}$ Recent work with STM (scanning tunneling microscopy) and SFM (scanning force microscopy) showed that it is possible to image electron flow in a 2D electron gas ${ }^{13}$ and a QD. ${ }^{14}$ We propose to use these techniques to image vortices in QDs.

In the context of a mesoscopic superconductor, vortices can be seen as an electromagnetic whirlpool through which the magnetic field penetrates the sample. One finds vortices in bulk superconducting materials (only in type-II supercon- 
ductors) as well as in mesoscopic superconductors. Since superconductivity is destroyed where the magnetic field penetrates the sample, vortices moving through the sample (e.g., because of a varying magnetic field) lead to dissipation and are therefore a serious problem limiting commercial applications. The critical parameters for superconductivity are determined by the vortex pattern and this pattern is determined by the geometry of the sample. ${ }^{8,15-17}$ This opens the possibility to manipulate the critical parameters, which in turn becomes important when we are interested in using superconductors as components, e.g., for cryoelectronics.

In pioneering work by Chibotaru and co-workers ${ }^{8-10}$ it was found that the vortex patterns which are formed have the same symmetry as the sample. In order to realize this they found the surprising result that for certain ranges of the magnetic field antivortices are introduced into the system. Their approach was based on an analytical gauge transformation for the vector potential which can only be realized for regular polygons. Here we follow a very different approach in which we solve the 2D differential equation using the finite difference technique. This approach gives us the flexibility to consider arbitrary geometries for the sample boundary and also arbitrary boundary conditions for the wave function.

The paper is organized as follows. In Sec. II we discuss the differential equation which has to be solved and the numerical procedure. Section III then presents the results for the QD with different confinements (square, rectangle, and triangle), while Sec. IV discusses the results for the superconducting films, wires, and thin superconducting squares near the superconducting/normal phase boundary. Finally, Sec. V sums up the most important conclusions.

\section{THEORY}

First we discuss the theoretical framework of the QD. The Hamiltonian for a single electron in a 2D QD, placed in a magnetic field is given by

$$
H=\left(\boldsymbol{p}+\frac{e}{c} \boldsymbol{A}\right) \frac{1}{2 m(x, y)}\left(\boldsymbol{p}+\frac{e}{c} \boldsymbol{A}\right)+V(x, y),
$$

where $\boldsymbol{A}$ is the vector potential corresponding to the perpendicular applied magnetic field $\boldsymbol{B}$ and $V(x, y)$ is the confinement potential of the 2D QD. Equation (1) must be solved using Dirichlet boundary conditions. The calculated energy will be given in units of $E_{0}=\hbar^{2} / 2 \mathrm{mS}$ as function of the flux $\phi / \phi_{0}=B S /(h c /|e|)$ through the surface area $S$ of the QD.

Second, we study mesoscopic superconductors. The linearized GL equation describes a superconductor near the superconducting-normal state phase transition and is given by

$$
\frac{1}{2 m^{*}}\left(\frac{\hbar}{i} \boldsymbol{\nabla}+\frac{e^{*}}{c} \boldsymbol{A}\right)^{2} \Psi=-\alpha(T) \Psi,
$$

where $m^{*}=2 m_{e}, e^{*}=2 e$, and $\alpha(T)$ is a temperature-dependent constant. The complex order parameter $\Psi(\boldsymbol{r})$ determines the number of Cooper pairs through $|\Psi(\boldsymbol{r})|^{2}=n_{s}(\boldsymbol{r})$ which is the density of Cooper pairs in the superconductor. The complete GL equation has an extra nonlinear term proportional to $|\Psi|^{2}$ which can be neglected at the superconducting-normal phase boundary. Notice that the linearized form of the GL equation is the regular Schrödinger equation for a free particle in which $-\alpha(T)$ plays the role of the eigenenergy.

The general boundary condition for superconductors, found by de Gennes, ${ }^{18}$ is given by

$$
\boldsymbol{n}\left(-i \hbar \nabla+\frac{e^{*}}{c} \boldsymbol{A}\right) \Psi=-i \hbar \beta \Psi,
$$

where $\boldsymbol{n}$ is the unity vector perpendicular to the surface of the superconductor and $\beta$ is a real number. This parameter $\beta$ determines the boundary conditions:

(1) A superconductor/vacuum (or superconductor/ insulator) transition is realized for $\beta=0$, which means that no superconducting current can flow out of the superconductor.

(2) $\beta<0$ describes a superconductor/metal interface, which will cause superconductivity to be weaker at the edges of the sample. For $\beta \rightarrow-\infty$ this condition reduces to the Dirichlet boundary condition for a QD, which is also realized at a superconductor/ ferromagnet interface (superconductivity will be strongly diminished at the edges of the sample).

(3) $\beta>0$ enhances superconductivity, which can be achieved experimentally by putting the mesoscopic superconductor in contact with another superconductor with a higher critical temperature $T_{c}$ or through a special treatment of the surfaces of the superconductor.

Previous publications in this area for fixed finite values of the parameter $\beta$ are Refs. 8, 19, and 20.

We numerically calculate the eigenvalues for both the QD and the mesoscopic superconductor. Apart from the spectrum, we are specifically interested in the vortices in both systems. To discuss the vorticity in a QD, we borrow an aspect that is used in the physics of mesoscopic superconductors and can be determined for both systems: the vorticity (also called "winding") number is defined by ${ }^{18}$

$$
l=\frac{1}{2 \pi} \oint_{C} \boldsymbol{\nabla} \theta \cdot d s,
$$

where $C$ is the contour along the boundary of the sample and $\theta$ is the phase of the wave function $\Psi=|\Psi| e^{i \theta}$. The vorticity number is directly related to the expectation value of the angular momentum operator $L_{z}$, as for an eigenstate of a given vorticity $l$ the leading angular dependence in the decomposition of the wave function is $\exp (-i l \theta)$. Vortices correspond to circular current patterns through $\boldsymbol{j}(\boldsymbol{r}) \sim \nabla \theta(\boldsymbol{r})$.

We solved the Schrödinger equation numerically using the finite difference technique (for the QDs) and the method of the finite elements, implemented in FEMLAB (for the mesoscopic superconductor in a magnetic field). ${ }^{21}$ To calculate eigenvalues and eigenvectors, we used ARPACK, ${ }^{22}$ a routine based on the Lanczos algorithm.

The results for the QD in Sec. III A recover the results found by Chibotaru et al. ${ }^{10}$ for the energy and vorticity of electrons in a square confinement. Here, we extend those results to a finite height confinement potential and to trian- 
gular and rectangular shaped QDs. The technique used in Ref. 10 is limited to specific geometries for the QD and to hard wall confinement. Chibotaru et al. ${ }^{8}$ also studied the linearized GL equation for mesoscopic superconductors, but only for the case $\beta=0$. Our approach is more general and allows arbitrary values of $\beta$ and arbitrary shapes of the mesoscopic superconductor.

\section{DIRICHLET BOUNDARY CONDITIONS (QUANTUM DOT)}

Because the energy spectrum, the vortex pattern and other calculated values of the confined electron obtained from Eq. (1) will be strongly influenced by the exact geometry, we consider different shapes for $V(x, y)$. We start with a square well and investigate the influence of the depth of the well on the energy spectrum and the vortex pattern. Next the symmetry of the confinement potential on the vortex pattern is investigated by implementing infinite high rectangular and triangular wells.

\section{A. Infinite square well}

Figure 1(a) shows the energy spectrum of a square well with hard wall confinement as function of the magnetic field $\phi / \phi_{0}$, where $\phi=B S$ is the flux through the square, $S=W^{2}$, and $W$ the width of the square. For comparison, the spectrum of a circular well (dashed curves) with the same surface area is also shown. The energy spectrum (in units of $E_{0}=\hbar^{2} / 2 m S$ ) of a circular well with hard wall confinement can be calculated semi-analytically ${ }^{23,24}$ and exhibits many more crossings than the spectrum of the square well. The latter can only be obtained numerically. The energy spectrum of the square well exhibits several anticrossings as a result of the mixing of different angular momentum states.

In a circular well the vorticity number is just the rotational quantum number of the corresponding eigenstate $\left(\left\langle L_{z}\right\rangle / \hbar\right.$ is a good quantum number), which is no longer the case in a square (and other geometries) because $\left[H, L_{z}\right] \neq 0$. As a consequence $\left\langle L_{z}\right\rangle$ is no longer quantized and it varies continuously for the square well as the magnetic field varies [see Fig. 1(b)]. As $\left\langle L_{z}\right\rangle / \hbar$ is related to the vorticity number $l$ it implies that vortices or antivortices will have to enter/exit the sample to obtain lower or higher values of $\left\langle L_{z}\right\rangle$. Notice from Fig. 1(a) that parts of the energy spectrum of the disk match the spectrum of the square dot and therefore we expect to find for these matching parts the same result for $\left\langle L_{z}\right\rangle / \hbar$ and $l$, respectively, as is indeed the case [see Fig. 1(b)]. Near avoided crossings, $\left\langle L_{z}\right\rangle / \hbar$ changes rapidly with the magnetic field to the $l$ value of the corresponding circular disk energy levels.

To investigate the changes in the vortex pattern, Fig. 2 shows vector plots of the gradient of the phase of the wave function at different values of the magnetic field using the wave functions that correspond to the energies indicated by the dots in Fig. 3. The images shown in Figs. 2(a)-2(c) clearly demonstrate the transition from a total $l$ value of 4 to $l=0$ for the upper energy level of the anticrossing: the transition is made by creating a vortex-antivortex pair at the

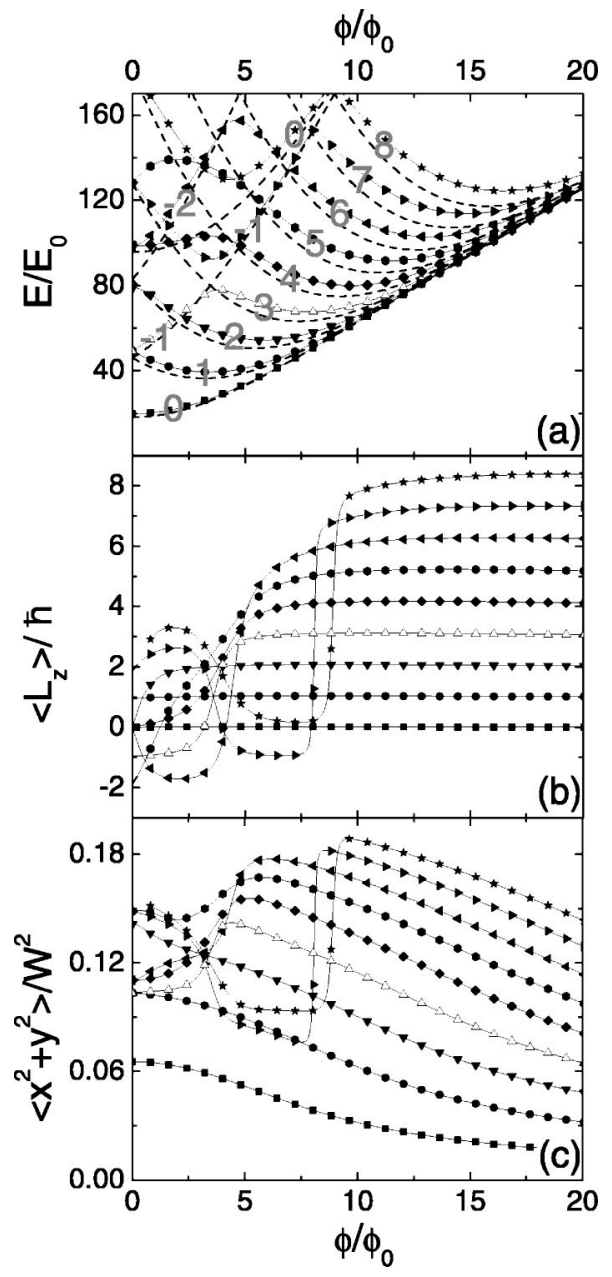

FIG. 1. (a) Energy spectrum of an electron in an infinite high square well (lines +symbol) and in an infinite high circular well (dashed lines), placed in a magnetic field $B$ perpendicular to the surface, as function of the flux $\phi=B S$ through the surface $S$ of the square. $\phi_{0}$ denotes the elementary flux quantum $h c /|e|$ and $E_{0}=\hbar^{2} / 2 m S$. The numbers indicated on the spectrum are the $l$ quantum numbers for the circular disk. (b) Expectation value of the angular momentum operator $L_{z}$ as function of the flux (magnetic field). (c) Expectation value $\left\langle x^{2}+y^{2}\right\rangle$ as function of the flux (magnetic field). The same type of symbols are used in all three figures for curves corresponding to the same energy level.

corners of a square and subsequently four vortices move toward the corners of the well and disappear (see also Ref. 10). For the lower energy level [Figs. 2(d)-2(f)] the opposite occurs: there is a transition from $l=0$ to $l=4$ by the entry of four vortices through the center of the edges of the square well. These four vortices nucleate with four antivortices which were already present in the system, so the $l$ value of the remaining vortices adds up to 4 . The disappearance of these four vortex-antivortex pairs is even more clearly illustrated in Fig. 4.

The positions of one pair of vortices-the vortex (vortex penetrating the sample) and the antivortex (antivortex present in the sample) — clearly demonstrates that for an increasing magnetic field: (i) an extra vortex appears, (ii) the vortices move toward each other, and (iii) the vortex- 


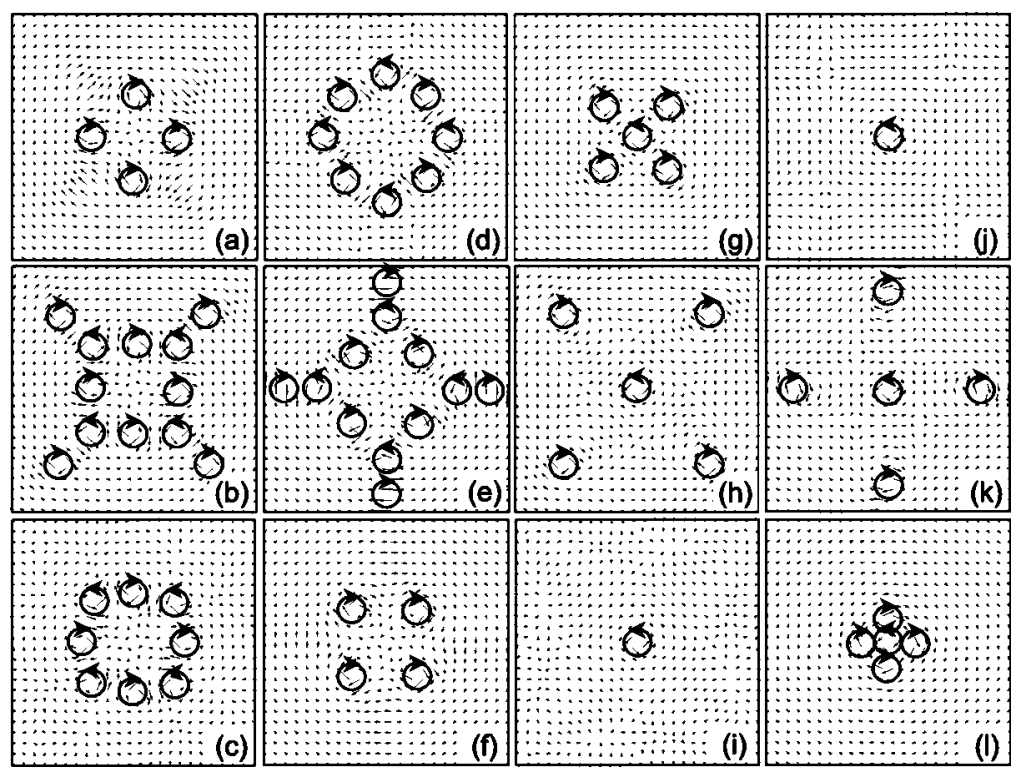

FIG. 2. Vortex patterns in (a)-(1) for the infinite square well. A clockwise current flow (i.e., a vortex) corresponds to $l=+1$, while $l=-1$ corresponds to a counterclockwise current flow (i.e. an antivortex). antivortex pair disappears. Notice that the vortex configurations of Figs. 2(a) and 2(f) belong to the same circular disk level $l=4$, but the vortex configurations are rotated over $45^{\circ}$ with respect to each other. Similarly the vortex positions for the other vortex configurations-before and after the anticrossing-belonging to the same disk level are rotated by $\phi=45^{\circ}$.

Similar results can be seen for other anticrossings: vortices and antivortices are created in a way that the pattern always reflects the square geometry. Another example depicted in Figs. 2(g)-2(1) shows the transition from $l=-1$ to $l=3$ and vice versa. The exit of the vortices happens again through the corners [Figs. 2(g) and 2(h)], leaving one antivortex $(l=-1)$ in the middle [Fig. 2(i)], while the entry of four vortices occurs through the edges of the sample [Fig. $2(\mathrm{k})$ ], creating a total $l$ value of 3 [Fig. 2(i)].

Since the wave function has to be zero at the location of the vortex (a vortex contains a phase singularity), the penetration or expulsion of vortices implies that the wave function also has to change continuously in order to let the vortex pattern be modified. The expectation value $\left\langle x^{2}+y^{2}\right\rangle$ as func-

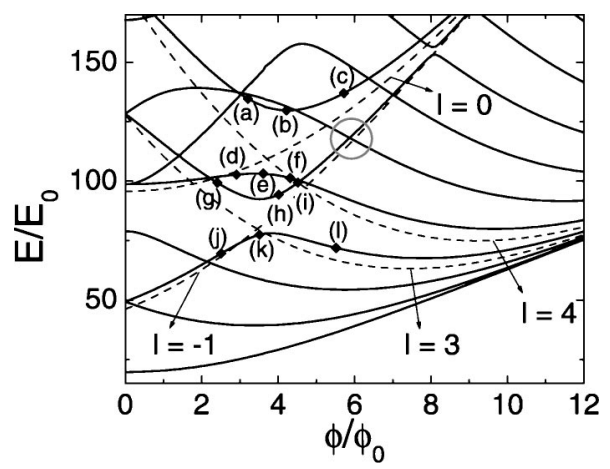

FIG. 3. Zoom of the energy spectrum of the square well (full lines) and the circular well (dashed lines). The current flow patterns (i.e., the vortex structure) in the points (a)-(1) are shown in Fig. 2. The gray circle highlights a crossing in the spectrum that will be discussed further in combination with Figs. 7-9. tion of the magnetic field in Fig. 1(c) shows this: it demonstrates how the wave function-despite an increasing magnetic field-can become more extended in order to let the vortices pass through the system. Only when the magnetic field is large enough such that the confinement of the electron becomes determined by the magnetic field instead of the square well, the value of $\left\langle L_{z}\right\rangle / \hbar$ will no longer change. The major changes in $\left\langle L_{z}\right\rangle / \hbar$ and $\left\langle x^{2}+y^{2}\right\rangle$ occur where the anticrossings in the spectrum are found.

\section{B. Finite square well}

The spectrum for the square well of finite height $V_{0} / E_{0}$ $=100$ is shown in Fig. 5. It appears as a scaled version of Fig. 1(a) for the infinite well. In Fig. 5 the energy is lower (because the particle is less confined than in the infinite well) and the anticrossings are located at smaller magnetic field values because the magnetic confinement will dominate the potential confinement for lower magnetic fields. We can con-

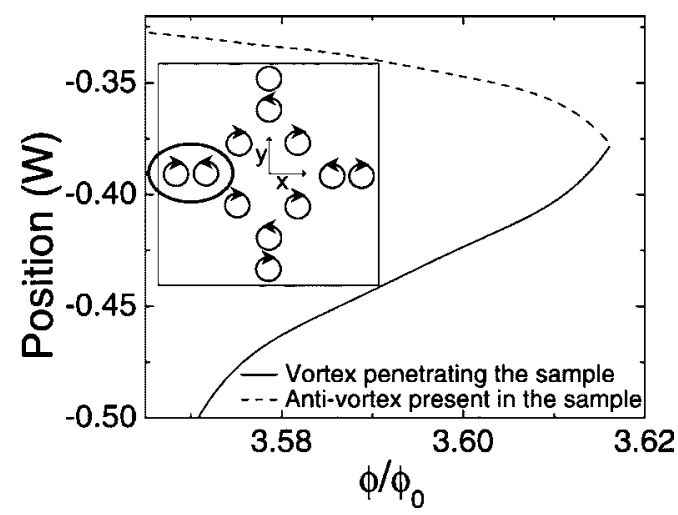

FIG. 4. Position of a vortex-antivortex pair as function of the flux (magnetic field). The inset schematically shows the vortex pattern and the set of vortices (circled pair) that was used to determine the position in the $x$ direction (the $y$ coordinate does not change in that direction). $W$ is the side length of the square. 


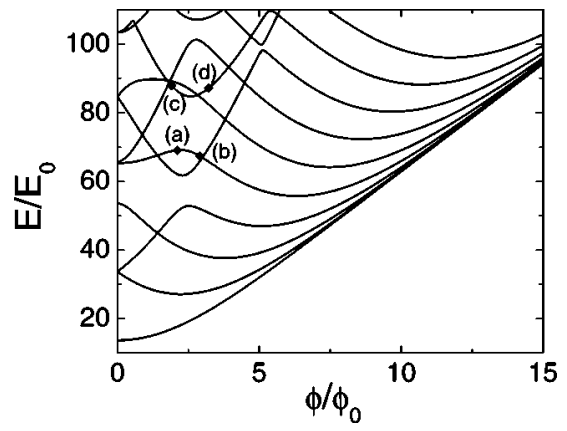

FIG. 5. Energy spectrum for an electron in a finite height square well $\left(V_{0} / E_{0}=100\right)$ as function of the flux $\phi=B S$ through the surface $S$ of the well. Note that $E_{0}=\hbar^{2} / 2 m S$ and $\phi_{0}=h c /|e|$.

clude that the height of the barrier does not fundamentally change the behavior of the energy spectrum.

Because now the electron wave function is different from zero in the barrier it allows the appearance of vortices or antivortices in the barrier region. Thus vortices will nucleate in the barrier material which is very different from the infinite well case where they can only nucleate at the edge of the sample. This new behavior is illustrated in Fig. 6 [compare with Figs. 2(a)-2(f)]. But once the vortices enter the well region their qualitative behavior is similar to the one we found for the infinite well case.

\section{Infinite rectangular well}

In the previous paragraphs we focused on systems with a $C_{4}$ symmetry (squares). If we switch to rectangular wells we reduce the symmetry of the confinement further. We consider a system with surface area $W \times W(1-\delta)$, where the parameter $\delta$ defines the aspect ratio of the two sides of the rectangle. This reduced symmetry will influence the number of anticrossings in the energy spectrum, which can be seen clearly in Fig. 7. It shows how a pair of energy levels that forms a crossing when the system is still a square $(\delta=0$, see gray circle in Fig. 3) changes to an anticrossing when the symmetry of the system becomes rectangular $(\delta \neq 0$, see gray circle in Fig. 8). The size of the level splitting in the anticrossing shown in Fig. 7 can be fitted to

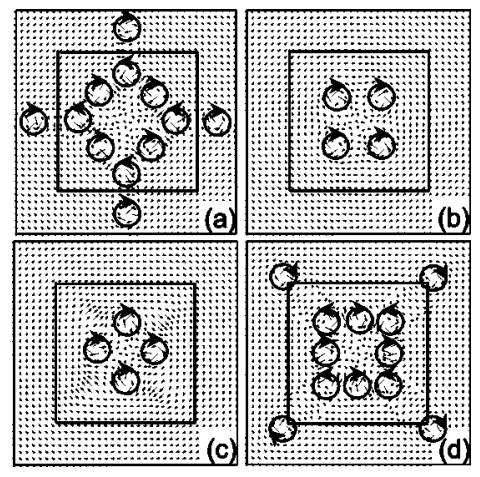

FIG. 6. Vortex patterns in (a)-(d) for the finite height square well. The wave functions that correspond to the points indicated in Fig. 5 were used to make these plots. The square indicates the boundary of the well.

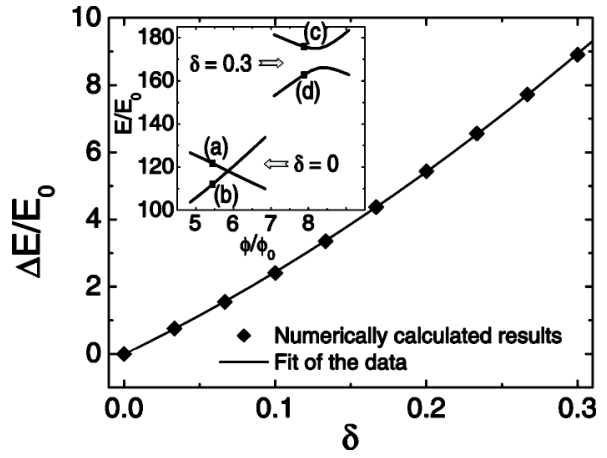

FIG. 7. Energy difference between a pair of energy levels for different values of $\delta$. Inset: detail of the studied pair of energy levels at different values of $\delta$. The complete spectrum for $\delta=0$ and $\delta=0.3$ can be found in Figs. 3 and 8 , respectively, with a gray circle indicating the studied crossing/anticrossing.

$$
\Delta E / E_{0}=a \delta^{2}+b \delta+c,
$$

where $a=26.69 \pm 0.92, b=21.81 \pm 0.29$, and $c=-0.01 \pm 0.02$. Since the system with reduced symmetry shows more anticrossings, we also expect a reduction in the symmetry of the density. The inset of Fig. 7 shows a detail of two energy spectra for different values of $\delta$ and we use these two cases to study the density of both systems (see Fig. 9). The letters (a)-(d) in the inset in Fig. 7 indicate the corresponding wave functions that were used to calculate the density, which is shown in Fig. 9. It is clear that by changing the square to a rectangular, the wave functions are not just squeezed into the new geometry, but they change fundamentally: even the number of maxima, zeros, and nodal lines change. In Fig. 9(a), we find 4 maxima and 5 zeros in the density arranged along the diagonals of the square. This configuration changes into 4 maxima along the diagonals of the rectangle, 4 zeros arranged in a rectangle, and a line of zero probability at $y=0[$ Fig 9(c) ]. For the lower branch (see inset of Fig. 7) the density consist of 4 maxima along the $x=0$ and $y=0$ lines and one zero in the center [Fig. 9(b)]. When the aspect ratio is $\delta=0.3$, this zero stays in the center [Fig. 9(d)], but now we only find maxima along the $y=0$ line and 4 submaxima on the diagonal of the rectangle.

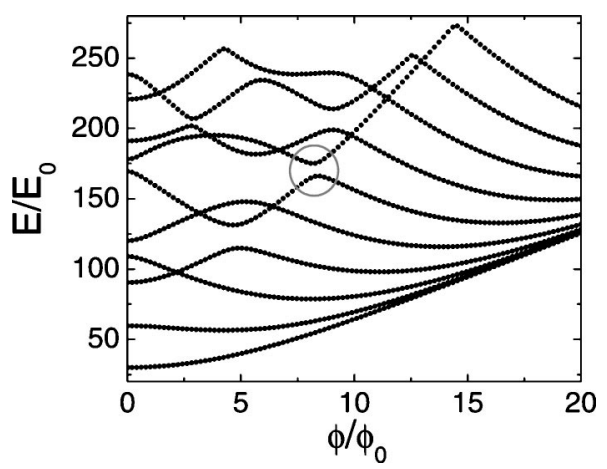

FIG. 8. Energy spectrum for an electron confined in a rectangular well $(\delta=0.3)$. The gray circle indicates the anticrossing that was studied in Fig. 7. 

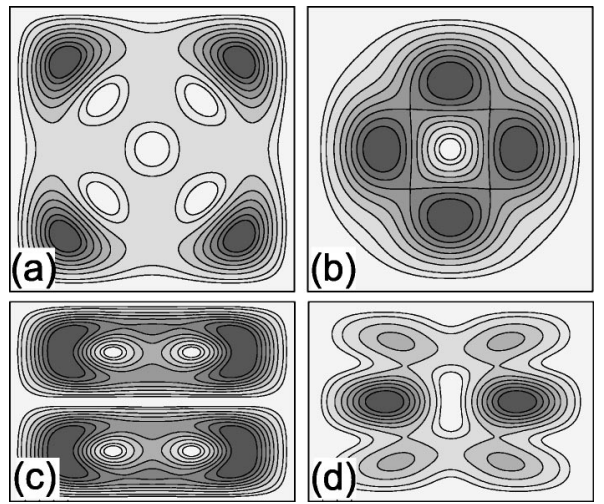

FIG. 9. Probability of the electron corresponding to points (a)(d), which are indicated in the inset of Fig. 7. High (low) density is given by the dark (light) regions.

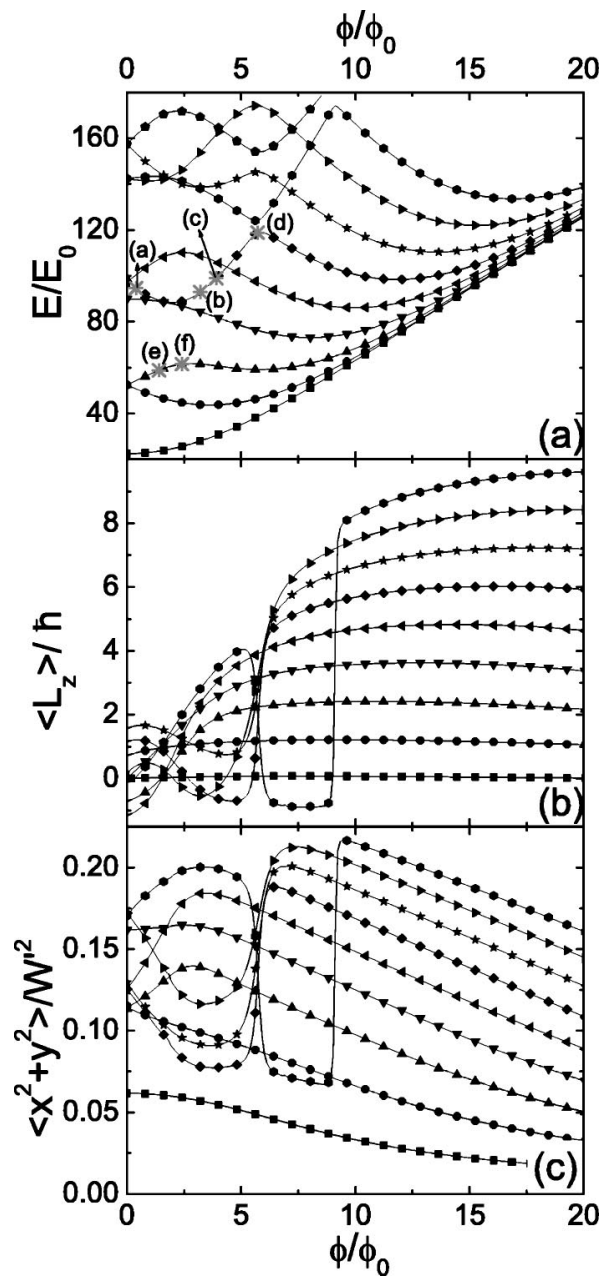

FIG. 10. (a) Energy spectrum of an electron in an infinite high triangular well placed in a magnetic field $B$ perpendicular to the surface, as function of the flux $\phi=B S$ through the surface $S$ of the triangle. $\phi_{0}$ denotes the elementary flux quantum $h c /|e|$ and $E_{0}=\hbar^{2} / 2 m S$. (b) Expectation value of the angular momentum operator $L_{z}$ as function of the flux (magnetic field). (c) Expectation value $\left\langle x^{2}+y^{2}\right\rangle$ as function of the flux (magnetic field). $W^{\prime}=2 / \sqrt[4]{3} W$, where $W$ is the length of a side of the triangle. The same type of symbols are used in all three figures for curves corresponding to the same energy level.

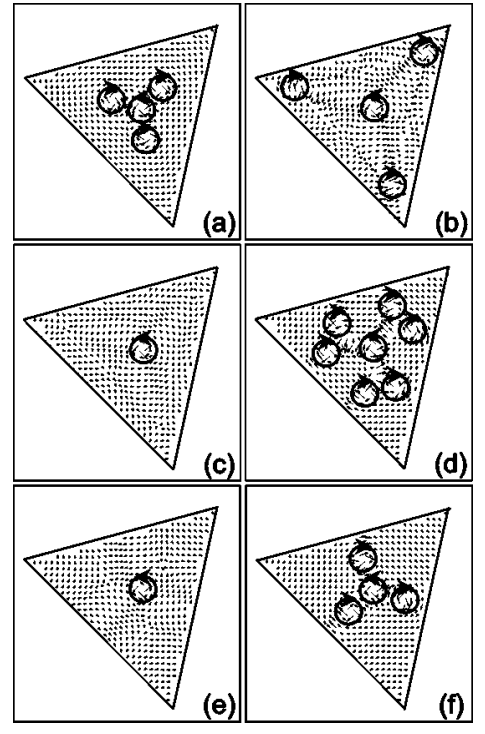

FIG. 11. Vortex patterns in points (a)-(f) indicated in Fig. 10(a) for the triangular well.

This sensitivity of the wave function on the geometry of the sample is similar to what was recently found for the single-particle Kohn-Sham orbitals in a density functional theory study of rectangular QDs with few electrons in a magnetic field. ${ }^{25}$

\section{Infinite triangular well}

Another interesting case is the one with triangular symmetry. We consider an equilateral triangle of which the position of the mesh points are rotated with respect to the triangle in order to make sure that the position of the triangle does not influence the numerical results. We first focus on the energy spectrum that shows more anticrossings than the spectrum of the square well as a consequence of the reduced symmetry [Fig. 10(a)]. As we discussed in Sec. III A, anticrossings correspond to a change of the expectation values $\left\langle L_{z}\right\rangle / \hbar$ and therefore the entry/exit of vortices in the system will take place at these anticrossings. Therefore the vortex pattern (Fig. 11) and the expectation values $\left\langle L_{z}\right\rangle / \hbar$ and $\left\langle x^{2}+y^{2}\right\rangle$ have also been calculated [Figs. 10(b) and 10(c)].

The wave functions corresponding to the indicated points (a)-(f) in Fig. 10(a) were used to determine the vortex pat-

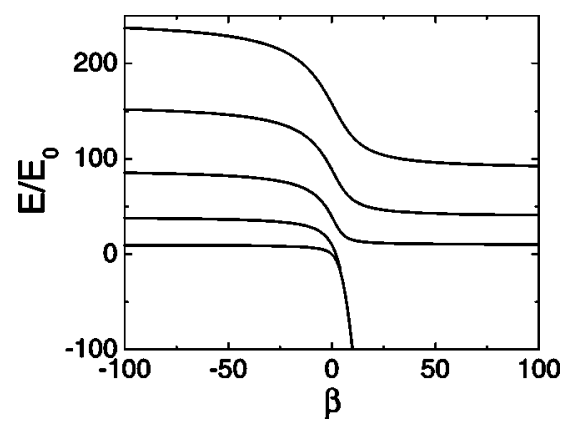

FIG. 12. The energy levels as function of $\beta$ (the parameter that determines the boundary condition). 

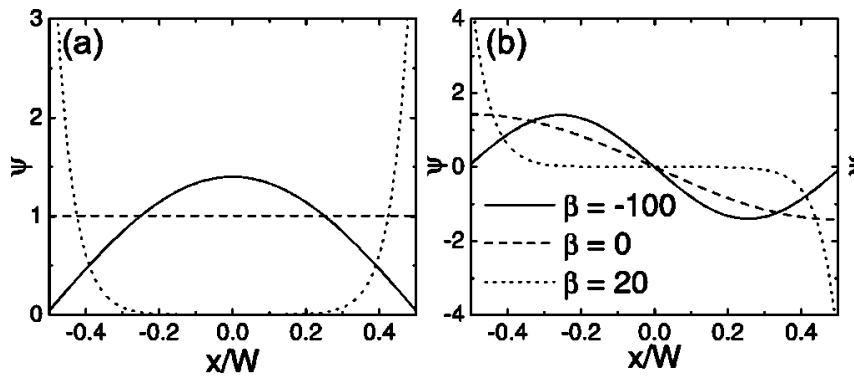

terns shown in Fig. 11. The initial number of vortices in Fig. 11(a) adds up to a total of $l=2$. Figure 10(b) indicates that the total $l$ value for that energy level will diminish as the magnetic field increases, which can be seen in Figs. 11(b) and 11(c): three vortices exit the sample through the corners of the triangle and leave one antivortex in the middle, $l=-1$. Further increasing the magnetic field we expect a strong increase of the $l$ value in point (d) [indicated in Fig. $10(\mathrm{a})]$, because of the strong rise of $\left\langle L_{z}\right\rangle / \hbar$ at $\phi / \phi_{0} \approx 6$. This is illustrated in Fig. 11(d) where 6 vortices enter through the sides of the triangle, bringing the total $l$ value to 5 . Beyond this point, no additional vortices enter or exit the sample anymore. As we expect, Figs. 11(e) and 11(f) of the lower energy level in Fig. 10(a) show the opposite situation of Figs. 11(b) and 11(c): in Fig. 11(e) we find one antivortex in the middle and as the magnetic field increases, three vortices enter through the edges of the sample, which results in a total of $l=2$. Notice that we find a remarkable $60^{\circ}$ rotation between the arrangement of exiting vortices in Figs. 11(a) and 11(b) as compared to the entry of vortices in Fig. 11(f). A similar phenomena was found for the square sample in Fig. 2. Also notice how the vortex pattern keeps the $120^{\circ}$ rotational symmetry in all figures, which is a consequence of the fact that the differential equation is linear. The latter implies that the wave function has the same symmetry as the confinement potential.

\section{VON NEUMANN BOUNDARY CONDITIONS}

\section{A. One-dimensional well with Von Neumann boundary conditions}

To investigate the influence of the parameter $\beta$ on the energy spectrum of a 2D system, it is instructive to solve first the Schrödinger equation for an electron in a onedimensional (1D) well with the general boundary condition $\boldsymbol{n} \cdot \boldsymbol{\nabla} \psi / \psi=\beta$, where $\boldsymbol{n}$ is the unity vector perpendicular to the edge of the sample. The spectrum is obtained from the transcendental equation

$$
\tan (k W)=\frac{2 \beta k}{\beta^{2}-k^{2}},
$$

where $k=\sqrt{2 m E / \hbar^{2}}$. This transcendental equation has to be solved numerically, but we discuss first some limiting cases.

(1) Limit $\beta \rightarrow-\infty: \tan (k W)=0 \Rightarrow E=\hbar^{2} \pi^{2} n^{2} / 2 m W^{2}$, which corresponds to the energy levels for a 1D infinite well. This case corresponds to the Dirichlet boundary conditions.

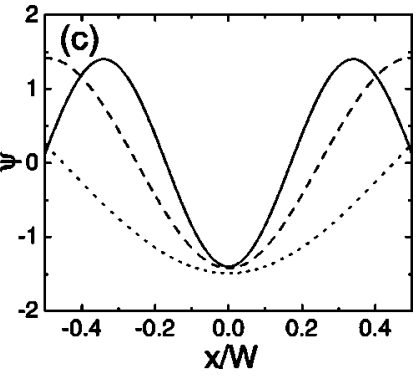

FIG. 13. Wave functions of the ground (a), the first (b), and the second (c) excited state for different values of $\beta$. W is the width of the well.
(2) Limit $\beta \rightarrow 0$ : This results in $\tan (k W)=0$, which gives the same result $E=\hbar^{2} \pi^{2} n^{2} / 2 m W^{2}$, but with the fundamental difference that $n=0$ is also a solution.

(3) $\beta>0$ : For positive $\beta$ values superconductivity is enhanced at the edges of the sample. Therefore it is possible to have $k=i \kappa$ and this yields

$$
\left(\beta^{2}+\kappa^{2}\right) \tanh (\kappa W)=2 \beta \kappa
$$

In the situation of large $\kappa W$ we find that $\beta^{2}+\kappa^{2}-2 \beta \kappa$ $=0 \Rightarrow k^{2}=-\beta^{2}$, which means that $E=-\hbar^{2} \beta^{2} / 2 m$ implying negative energy values which are not possible in the Dirichlet case. Note that Eq. (7) reduces to Eq. (4) of Ref. 19 for large $\kappa$ values.

(4) Limit $\beta \rightarrow \infty$ : We can also have $\tan (k W)=0 \Rightarrow E$ $=\hbar^{2} \pi^{2} n^{2} / 2 m W^{2}$ and these solutions are identical to the one for the Dirichlet boundary conditions.

The numerical solutions to Eq. (6) for the 5 lowest levels are given in Fig. 12 as function of $\beta$. All the limiting cases mentioned above can be found in the figure. Notice the remarkable behavior of the two lowest levels for $\beta>0$ which become degenerate and split off from the rest of the spectrum.

The wave functions of the ground state in Fig. 13(a) illustrate more clearly what happens when we change the boundary conditions. For $\beta \rightarrow-\infty$ the wave function has to be zero at the boundary of the well (full curve), and $\beta \rightarrow-\infty$ corresponds to the Dirichlet boundary conditions. For $\beta=0$ (dashed curve) the derivative of the wave function has to be zero at the edges. The lowest level wave function, which is zero at the edges for $\beta=-\infty$, evolves continuously to a constant function for $\beta=0$. For positive $\beta$ values, the wave function becomes strongly localized at the boundaries of the well

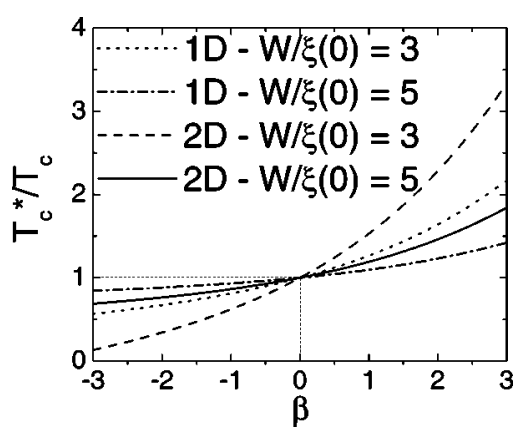

FIG. 14. Comparison of the effective critical temperature [for 1D (i.e., film) and 2D (i.e., square) confinement] with the critical temperature $T_{c}$ of the bulk superconductor as function of the boundary conditions, indicated by the parameter $\beta$. 

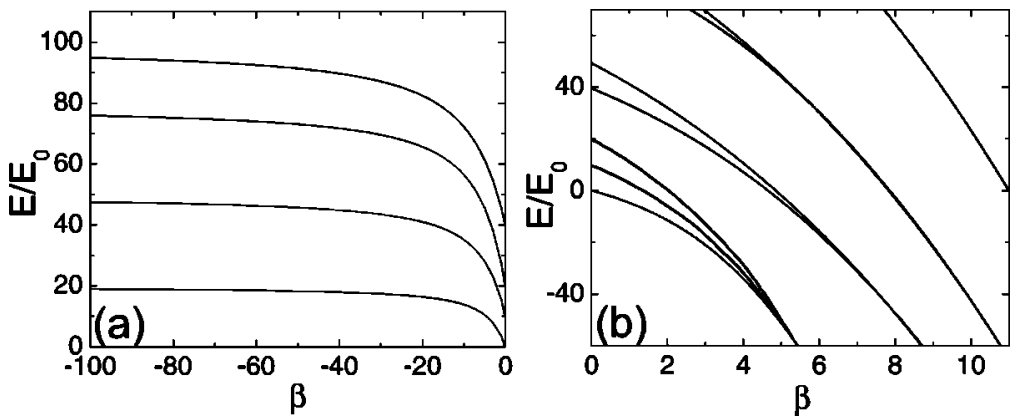

FIG. 15. Spectrum as function of the parameter $\beta$ for a square sample. Note that $E_{0}=\hbar^{2} / 2 m S$. (dotted curve). In Figs. 13(b) and 13(c) we show the wave function of the first two excited states. These figures make it physically understandable why for $\beta \gg 0$ : (i) the two lowest levels become degenerate, and (ii) why the second excited state has an energy which is equal to the ground state energy for $\beta \ll 0$.

The ground state energy level presented in Fig. 12 can be used in the context of superconductivity. In the linearized GL equation, $\alpha(T)$ corresponds to the energy we obtained in the 1D Schrödinger equation. We find the following relation between energy and critical temperature $T_{c}^{*}$ :

$$
\frac{E}{E_{0}}=\left(\frac{W}{\xi(0)}\right)^{2}\left(1-\frac{T_{c}^{*}}{T_{c}}\right) ; \quad E_{0}=\frac{\hbar^{2}}{2 m W^{2}} .
$$

Here we replaced $T$ by $T_{c}^{*}$, the effective critical temperature of the superconductor, because the linearized GL equation is valid exactly at the superconducting-normal phase boundary. This implies that the temperature $T$ corresponds to the effective critical temperature $T_{c}^{*}$. Using the ground state energy, which is the energy level of significance for superconductivity, we obtain $T_{c}^{*}$ shown in Fig. 14. The effective critical temperature $T_{c}^{*}$ of the mesoscopic sample is compared to the critical temperature $T_{c}$ in bulk material. Notice that for negative values of $\beta$ we find that $T_{c}^{*}<T_{c}$, thus superconductivity is reduced, while for positive $\beta$ values $T_{c}^{*}>T_{c}$, which indicates enhancement of superconductivity. These results are valid for superconducting films, where the confinement is one-dimensional.

\section{B. Two-dimensional square well with Von Neumann boundary conditions (no magnetic field)}

Since the 2D potential well (without an applied magnetic field) is equivalent to two decoupled 1D wells, we can use the results of the $1 \mathrm{D}$ well to obtain the results for the $2 \mathrm{D}$ system. Figure 15 shows the energy spectrum for the 2D well for (a) negative and (b) positive $\beta$ values. This spectrum can be directly obtained from the 1D case (Fig. 12) because of the separability of the variables: $E\left(n_{x}, n_{y}\right)=E_{n_{x}}+E_{n_{y}}$ where $E_{n_{i}}$ is the 1D energy level as depicted in Fig. 12 . The $\beta>0$ spectrum shown in Fig. 15(b) needs extra discussion. Each branch consists of 4 levels. The lowest branch is constructed from the states $\left(n_{x}, n_{y}\right)=(1,1),(1,2),(2,1),(2,2)$ where the middle two, i.e., $(1,2)$ and $(2,1)$, are degenerate. The next branch consists of two energy levels which are each twofold degenerate: $(1,3),(3,1),(2,3),(3,2)$.

Only the ground state of the energy spectrum is significant for the superconducting/normal boundary. Figure 14 shows the link between the temperature and the boundary conditions (parameter $\beta$ ). Notice that there are two different cases: negative values of $\beta$ corresponding to $T_{c}^{*}<T_{c}$, thus superconductivity is reduced, while for $\beta>0$ we have $T_{c}^{*}>T_{c}$, which implies enhancement of superconductivity. For 2D confinement the effect of the parameter $\beta$ on $T_{c}^{*}$ is larger than in $1 \mathrm{D}$, i.e., superconducting film, case, which was already previously demonstrated in Refs. 19 and 20.

\section{Two-dimensional square well with Von Neumann boundary conditions (with magnetic field)}

Adding a magnetic field makes the situation a lot more complicated and forces us to use numerical techniques to calculate the energy spectrum. Our numerical approach enables us to calculate the energy spectrum as function of the applied magnetic field for any value of $\beta$. A similar study for circular wires was presented in Ref. 26. Figure 16 shows the result of the numerical calculations and displays the energy spectrum for a positive $(\beta=1)$ and a negative $(\beta=-1.25)$ value of $\beta$. The ground state in these figures shows an oscillating behavior. If we link the energy to the parameter $\alpha(T)$,
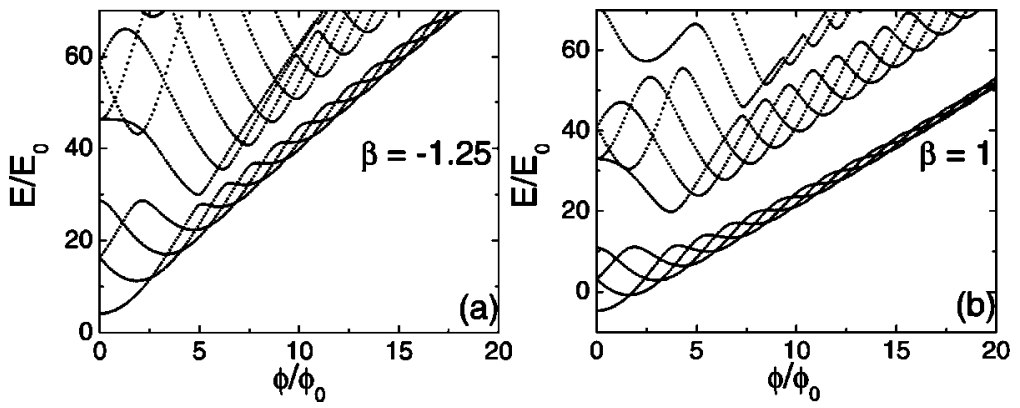

FIG. 16. Spectrum as function of the flux $\phi$ through the surface $S$ of the square well for (a) $\beta=-1.25$ and (b) $\beta=1$. The units are $E_{0}=\hbar^{2} / 2 m S$ and $\phi_{0}=h c /|e|$. 


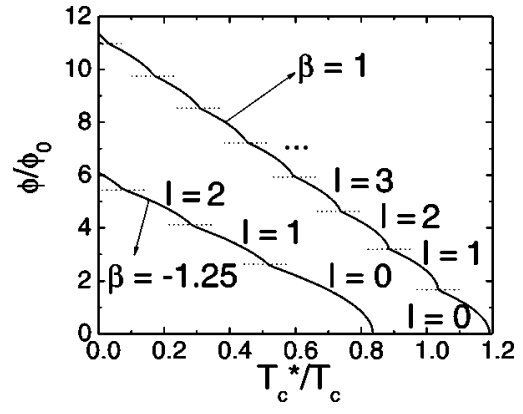

FIG. 17. Superconducting-normal state phase boundary for $\beta=-1.25$ (left curve) and $\beta=1$ (right curve) for the case of a square with width $W / \xi(0)=5$.

we find oscillations in the superconducting-normal phase boundary (Fig. 17). At zero magnetic field we can already see that $\beta=1$ corresponds to enhanced superconductivity since $T_{c}^{*} / T_{c}>1$, while $\beta=-1.25$ clearly illustrates that these boundary conditions cause a reduced effect on superconductivity. By calculating the expectation value of the angular momentum operator $L_{z}$ for this system, we find that every oscillation corresponds to the entry of a vortex ( $l$ value indicated in Fig. 17): as the magnetic field increases, vortices gradually penetrate the sample and will eventually destroy superconductivity, no matter how low the temperature.

The phase diagram for an arbitrary value of $\beta$ is shown in Fig. 18, where the number of vortices at the S/N boundary in a superconducting square are indicated. Alternatively, we can divide the plane into a part where the sample is superconducting and a part where it is in the normal phase which is shown by the dashed line in Fig. 18. The dashed line which separates both regions depends on the size of the sample. In Fig. 18 we took $W / \xi(0)=5$. The current patterns that correspond to the $l$ values in Fig. 18 are shown in Fig. 19. For $l=2$ and $l=6$, a double quantized vortex, i.e., a giant vortex ${ }^{27}$ appears in the center of the sample. Such giant vortices were recently experimentally observed in Ref. 28. For a given $l$ value only a single pattern is found at the $\mathrm{S} / \mathrm{N}$ boundary, but the pattern becomes more compact as $\beta$ decreases. Figure 19 shows how the symmetry of the square is preserved in the vortex pattern. For $l=3$ and $l=7$ this can be realized by adding antivortices. Such geometry induced antivortices were predicted in Ref. 8 but have, up to now, not been observed experimentally. A probable reason ${ }^{17}$ is their

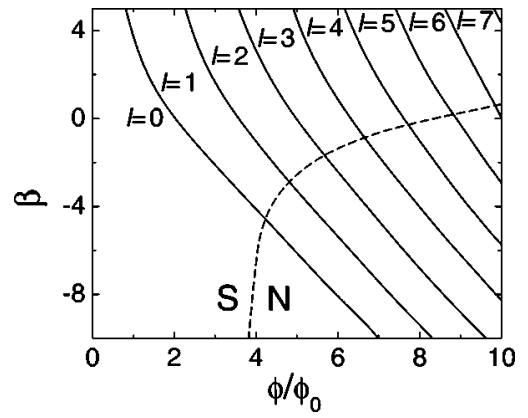

FIG. 18. Phase diagram for the vorticity of a mesoscopic superconductor at the $\mathrm{S} / \mathrm{N}$ boundary. $\beta$ is the parameter determining the boundary conditions, $\phi=B S$ and $\phi_{0}$ is the elementary flux quantum. The dashed line separates the superconducting phase $(\mathrm{S})$ with the normal phase $(\mathrm{N})$ and is drawn for $W / \xi(0)=5$.

small stability region when one moves away from the $\mathrm{S} / \mathrm{N}$ boundary. Notice that by decreasing $\beta$, the ground state evolves to a state that contains no vortices $(l=0)$, which is the case for the ground state of an electron in a QD that is found for $\beta \rightarrow-\infty$.

\section{CONCLUSIONS}

We found that confinement and boundary conditions play an important role in the properties of mesoscopic samples. It strongly influences the vortex pattern and the eigenenergies.

The vorticity number, the expectation value of the angular momentum operator $L_{z}$ and the energy spectrum are closely related to each other for all the studied cases of QDs. The energy spectrum of the QD exhibits more anticrossings as the symmetry is reduced, this is translated into a step in the value of $\left\langle L_{z}\right\rangle / \hbar$ which in turn corresponds to a change of the vorticity number $l$. Therefore, fundamental changes in the vortex pattern occur for lower symmetry samples with variation of the magnetic field. The vortex pattern undergoes changes near every anticrossing of energy levels. By changing the magnetic field, vortices and antivortices are created in such a way that the symmetry of the sample is reflected in the vortex pattern as agrees with Ref. 10.

Furthermore, critical parameters for superconductors can be influenced by changing the boundary conditions and the applied magnetic field. For example, we found that the ef-

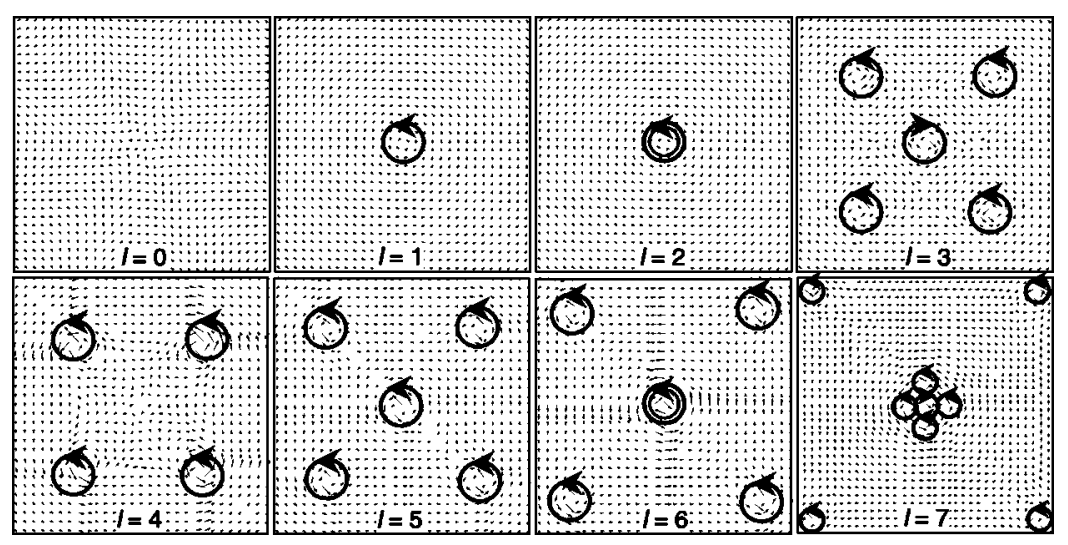

FIG. 19. Schematic vortex patterns for different values of $l$. Only a small part of the square with $W / \xi(0)=5$ near the vortex pattern is shown. A counterclockwise rotation corresponds to $l=1$, a clockwise rotation to $l=-1$ (Ref. 29). The double circle in the case of $l=2$ and $l=6$ indicates a giant vortex which caries two flux quanta. These patterns can be found in areas with corresponding $l$-value in Fig. 18. 
fective critical temperature of the sample depends on the boundary conditions and can be diminished (effective critical temperature lower than the bulk critical temperature) or enhanced (effective critical temperature larger than the bulk critical temperature). As function of the magnetic field the effective critical temperature at the $\mathrm{S} / \mathrm{N}$ boundary exhibits an oscillatory behavior reminiscent of the stepwise increase of vorticity in the sample. In order for the symmetry of the sample to be reflected in the vortex pattern, which is a consequence of the linearity of the GL equation, giant vortices and antivortices have to be added.

\section{ACKNOWLEDGMENT}

This work was supported by the Flemish Science Foundation (FWO-Vl).
*Electronic address: an.slachmuylders@ua.ac.be

†Electronic address: bart.partoens@ua.ac.be

Flectronic address: francois.peeters@ua.ac.be

${ }^{1}$ T. Ando, A. B. Fowler, and F. Stern, Rev. Mod. Phys. 54, 437 (1982).

${ }^{2}$ Z.-L. Ji and K.-F. Berggren, Phys. Rev. B 52, 1745 (1995).

${ }^{3}$ A. S. Sachrajda, R. Ketzmerick, C. Gould, Y. Feng, P. J. Kelly, A. Delage, and Z. Wasilewski, Phys. Rev. Lett. 80, 1948 (1998).

${ }^{4}$ S. M. Reimann and M. Manninen, Rev. Mod. Phys. 74, 1283 (2002).

${ }^{5}$ L. P. Gork'ov showed in 1959 [Zh. Eksp. Teor. Fiz. 36, 1918 (1959)] that the GL theory was, in fact, a limiting case of the BCS theory allowing one to treat in a more intuitive way the spatially inhomogeneous regime (regime in which superconducting and normal domains coexist).

${ }^{6} \mathrm{M}$. Tinkham, Introduction to Superconductivity (McGraw-Hill, New York, 1975).

${ }^{7}$ C. DeWitt, B. Dreyfus, and P. G. de Gennes, Low-temperature Physics (Gordon and Breach, New York, 1962).

${ }^{8}$ L. F. Chibotaru, A. Ceulemans, V. Bruyndoncx, and V. V. Moshchalkov, Nature (London) 408, 833 (2000).

${ }^{9}$ L. F. Chibotaru, A. Ceulemans, V. Bruyndoncx, and V. V. Moshchalkov, Phys. Rev. Lett. 86, 1323 (2001).

${ }^{10}$ L. F. Chibotaru, A. Ceulemans, M. Lorenzini, and V. V. Moshchalkov, Europhys. Lett. 63, 159 (2003); 63, 476 (2003).

${ }^{11}$ H. Saarikoski, A. Harju, M. J. Puska, and R. M. Nieminen, Phys. Rev. Lett. 93, 116802 (2004).

${ }^{12}$ M. B. Tavernier, E. Anisimovas, and F. M. Peeters, Phys. Rev. B 70, 155321 (2004).

${ }^{13}$ M. A. Topinka, B. J. LeRoy, R. M. Westervelt, S. E. J. Shaw, R. Fleischmann, E. J. Heller, K. D. Maranowski, and A. C. Gossard, Nature (London) 410, 183 (2001).

${ }^{14}$ A. Pioda, S. Kičin, T. Ihn, M. Sigrist, A. Fuhrer, K. Ensslin, A. Weichselbaum, S. E. Ulloa, M. Reinwald, and W. Wegscheider, Phys. Rev. Lett. 93, 216801 (2004).
${ }^{15}$ P. Kes, J. Aerts, and A. Troyanovski, Nature (London) 399, 665 (1999).

${ }^{16}$ A. K. Geim, I. V. Grigorieva, S. V. Dubonos, J. G. S. Lok, J. C. Moon, A. E. Filippov, and F. M. Peeters, Nature (London) 390, 259 (1997)

${ }^{17}$ B. J. Baelus and F. M. Peeters, Phys. Rev. B 65, 104515 (2002).

${ }^{18}$ P. G. de Gennes, Superconductivity of Metals and Alloys (Benjamin, New York, 1966).

${ }^{19}$ E. Montevecchi and J. O. Indekeu, Europhys. Lett. 51, 661 (2000).

${ }^{20}$ E. Montevecchi and J. O. Indekeu, Phys. Rev. B 62, 14359 (2000).

${ }^{21}$ S. S. Rao, Applied Numerical Methods for Engineers and Scientists (Prentice Hall, Englewood Cliffs, NJ, 2002).

${ }^{22} \mathrm{http}$ ///www.caam.rice.edu/software/ARPACK/

${ }^{23}$ The energy spectrum for the confined electron is given by $E_{n l}$ $=\hbar \omega_{c}\left(a_{n l}+(1+|l|-l) / 2\right)$, where $a_{n l}$ can be determined from the boundary condition ${ }_{1} F_{1}=\left(-a_{n l} ;|l|+1 ; \lambda R^{2}\right)=0$ with $\lambda=m \omega_{c} / 2 \hbar$ and $R$ the radius of the circular well. The energy levels can be degenerate in the $l$-quantum number.

${ }^{24}$ S. Flügge, Practical Quantum Mechanics (Springer, New York, 1974).

${ }^{25}$ E. Räsänen, A. Harju, M. J. Puska, and R. M. Nieminen, Phys. Rev. B 69, 165309 (2004).

${ }^{26}$ B. J. Baelus, S. V. Yampolskii, F. M. Peeters, E. Montevecchi, and J. O. Indekeu, Phys. Rev. B 65, 024510 (2002).

${ }^{27}$ V. A. Schweigert, F. M. Peeters, and P. S. Deo, Phys. Rev. Lett. 81, 2783 (1998).

${ }^{28}$ A. Kanda, B. J. Baelus, F. M. Peeters, K. Kadowaki, and Y. Ootuka, Phys. Rev. Lett. 93, 257002 (2004).

${ }^{29} \mathrm{We}$ hereby take the convention used in superconductivity. This is the opposite of the convention we used in the case of the Dirichlet boundary conditions and therefore corresponds to a change in the direction of the magnetic field. 